\title{
LOGICAL ANALYSIS OF THE BOHR COMPLEMENTARITY PRINCIPLE IN AFSHAR'S EXPERIMENT UNDER THE NAFL INTERPRETATION
}

\author{
RADHAKRISHNAN SRINIVASAN \\ IBM India Software Labs, Embassy Tech Zone, Building ETB1-5F-A, Phase 2, Rajiv Gandhi \\ Infotech Park, Hinjewadi, Pune 411057, India \\ sradhakr@in.ibm.com \\ Received 11 July 2005 \\ Revised 6 November 2008 and 26 January 2010
}

\begin{abstract}
The NAFL (non-Aristotelian finitary logic) interpretation of quantum mechanics requires that no 'physical' reality can be ascribed to the wave nature of the photon. The NAFL theory QM, formalizing quantum mechanics, treats the superposed state $(S)$ of a single photon taking two or more different paths at the same time as a logical contradiction that is formally unprovable in QM. Nevertheless, in a nonclassical NAFL model for QM in which the law of noncontradiction fails, $S$ has a meaningful metamathematical interpretation that the classical path information for the photon is not available. It is argued that the existence of an interference pattern does not logically amount to a proof of the self-interference of a single photon. This fact, when coupled with the temporal nature of NAFL truth, implies the logical validity of the retroactive assertion of the path information (and the logical superfluousness of the grid) in Afshar's experiment. The Bohr complementarity principle, when properly interpreted with the time dependence of logical truth taken into account, holds in Afshar's experiment. NAFL supports, but not demands, a metalogical reality for the particle nature of the photon even when the semantics of QM requires the state $S$.
\end{abstract}

Keywords: Bohr complementarity principle; Afshar's experiment; NAFL interpretation

\section{Introduction}

Shahriar S. Afshar $1|2| 3$ has recently performed a variant of Young's two-slit experiment, in which he claims to have demonstrated the falsification of the celebrated Bohr complementarity principle, and thereby, the Copenhagen interpretation of quantum mechanics. In Afshar's experiment, sketched in Fig. 1, a source emits photons towards a screen with two slits marked U and L. A suitably located converging lens downstream of the screen focuses the photon streams into sharp images of the two slits $\mathrm{U}$ and $\mathrm{L}$, marked $\mathrm{U}^{\prime}$ and $\mathrm{L}^{\prime}$ respectively, at location $\sigma_{2}$ (for simplicity, we do not distinguish between the image plane and the focal plane in Fig. 11). Afshar notes that these images serve to provide sharp which-way information for the photons, thereby confirming their particle status. A wire grid is placed at location $\sigma_{1}$ in Fig. 1, such that the (thin) wires occur at precisely the theoretically predicted minima of the interference pattern due to the superposed states of the 

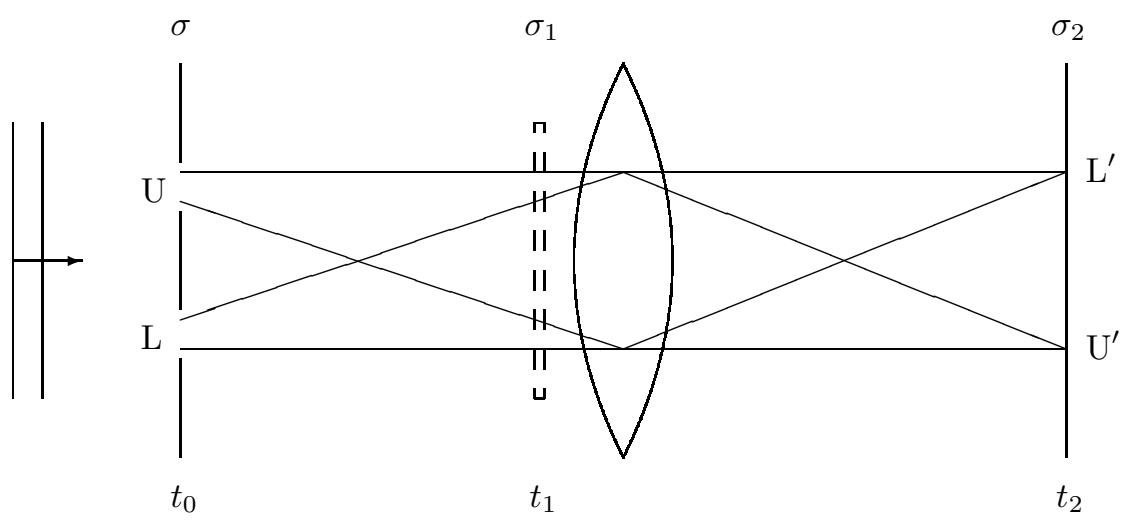

Fig. 1. Sketch of Afshar's experiment

photons. Afshar observes that the wire grid effectively performs a nondestructive confirmation of the superposition; when both slits are open, no distortion or reduction in intensity of the images at $\sigma_{2}$ is observed as compared to the case when the wire grid is removed. The expected distortion and reduction in intensity do occur when one of the slits is closed. This seemingly rules out the classical particle state of the photons between the locations $\sigma$ and $\sigma_{1}$ when both slits are open, and confirms their wave nature, since blocking of photons by the wire grid does not occur to the classically expected extent. Afshar concludes that both the sharp particle and wave natures of the photon are exhibited in a single experiment, in violation of the Bohr complementarity principle. In particular, Afshar 1 claims that the Englert-Greenberger duality relation 415 is falsified in his experiment.

Afshar's interpretation of his experiment has many critics (see Refs. 66 11), but there is no consensus on why Afshar is supposedly wrong. The most prominent of these critics seems to be Kastner 6 , who rejects the existence of which-way information in not only the Afshar experiment, but also in any classical two-slit experiment even when the wire grid is not present. Kastner's argument is essentially based on a realist interpretation in which the presence of an interference pattern requires the photon to 'really' pass through both slits. Kastner concludes that post-selection of the photon in a 'which-slit' basis does not imply that the photon passed through only one slit. In a subsequent article, Kastner 7 cites the two-slit experiment of Srikanth 12 as even more dramatic than the Afshar experiment, in that the existence of the interference pattern is irreversibly recorded (rather than just inferred) and yet post-selection of the photon in a 'which-slit' basis is possible The following passage from Kastner 7 clearly reveals her realist argument:

This is even more dramatic than the Afshar result because clearly $V=1$ since a fully articulated interference pattern has been irreversibly recorded

\footnotetext{
${ }^{a}$ An anonymous referee has rejected the validity of Srikanth's experiment
} 
- not just indicated indirectly - and yet a measurement can be performed after the fact that seems to reveal 'which slit' the photon went through. However, the point is that the detector's vibrational mode remains in a superposition until that measurement is made, implying that each photon indeed went through both slits. As Srikanth puts it, '...the amplitude contributions from both paths to the observation at [detector element] $x$ results in a superposition of vibrational modes. The initial superposition leaves behind a remnant superposition.' 12 . So, just because one can 'post-select' by measuring the vibrational observable and end up with a particular corresponding slit eigenstate doesn't mean the particle actually went through that slit; in a very concrete sense, it went through both slits.

Kastner $6 / 7$ concludes that the Bohr complementarity principle is not violated in the Afshar experiment and that the Englert-Greenberger duality relation is not applicable in all similar experiments in which an interference pattern can be inferred

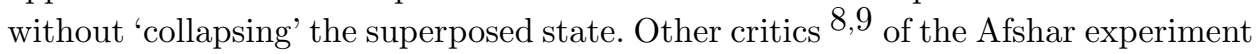
agree with Kastner that there is no which-way information and like Kastner, argue for a realist interpretation in which the photon passed through both slits. However, a few workers 1011 assert that while which-way information is present in the Afshar experiment, visibility of the fringes is very small because the interference pattern is not actually recorded in the Afshar experiment. This group rejects the inference of the existence of an interference pattern and insists that only an experimental recording of the same is acceptable. Afshar's co-workers ${ }^{13}$ and O'Hara ${ }^{14}$ support the Afshar interpretation that both the particle and wave properties of the photon co-exist in a real sense, thereby rejecting both the Bohr complementarity principle and the Englert-Greenberger duality relation.

It is the purpose of this paper to analyze the status of the Bohr complementarity principle in Afshar's experiment, particularly in the light of the non-Aristotelian finitary logic (NAFL) recently proposed by the author $15[16[17 \mid 18$. We will not concern ourselves with the nuts and bolts of Afshar's experiment in an attempt to evaluate his claims from the point of view of experimental physics. Instead, we consider the experiment from a purely logical angle, in the case when Afshar's claims are granted as experimentally sound. It will be seen that the NAFL interpretation of the quantum superposition principle does indeed uphold complementarity, despite the co-existence of the interference pattern and the path information for the photons that arrive at the image plane. Indeed, we argue that the interference pattern should, in principle, be reconstructable for these photons from just the path information, without any need for the controversial grid in Afshar's experiment. In fact the grid is not only logically superfluous, but also does not provide a complete reconstruction of the interference pattern, as would be possible from analysis of the

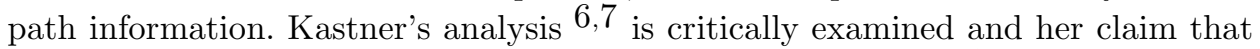
Cramer's Transactional Interpretation of quantum mechanics 19 rescues the complementarity principle in Afshar's experiment is disputed. What the experiments of 
Afshar and others really establish is that any realist interpretation of quantum mechanics is highly problematic from a physical and philosophical point of view. Both the Bohr complementarity principle and the Englert-Greenberger duality relation (at its extreme ends) are upheld in the Afshar experiment when they are properly interpreted to take into account the time dependence of logical truth, as embodied by NAFL. The NAFL interpretation really vindicates the original non-realist Copenhagen interpretation championed by Neils Bohr and others, which, contrary to Afshar's claims, remains unscathed by the Afshar experiment. While the Copenhagen interpretation formulates Bohr complementarity as a physical principle, the NAFL interpretation enshrines it as a sacred and inviolable logical principle which follows from basic postulates that embody finitary reasoning.

\section{Summary of the main argument and conclusions}

This section highlights the main argument and conclusions of this paper; the details are presented in subsequent sections. Consider the modified experiment of Afshar 3 , which was originally proposed by Wheeler 20 and is described in Sec. 5 of Ref. 13 , as quoted below.

... a laser beam impinges on a 50:50 beam splitter and produces two spatially separated coherent beams of equal intensity. The beams overlap at some distance, where they form an interference pattern of bright and dark fringes. At the center of the dark fringes we place thin wires. ... Beyond the region of overlap the two beams fully separate again. There, two detectors are positioned such that detector $1^{\prime}$ detects only the photons originating from mirror 1 , and detector $2^{\prime}$ detects only photons originating from the beam splitter (mirror 2). Since the pathway of the photon is practically unobstructed, a study of the electric fields involved together with conservation of momentum allows us to uniquely identify, with high probability, the respective mirror as the place where that photon originated.

The wire grid in the modified experiment plays the same role as in the original Afshar experiment and Afshar 3 concludes that the results of the two experiments are in agreement.

The above experiment is similar in essence to the quantum eraser, the delayedchoice version of which is particularly interesting 21. When the two photon beams overlap, they contain interference information and when they separate, they contain which-way information. As long as no measurements are made, the available information may be reversed as many times as one chooses. The key point here is that when the photon beams provide interference information, there is no way to extract which-way information and vice versa; this is essentially a formulation of the Bohr complementarity principle and is fully in agreement with the Copenhagen 
interpretation. $\mathrm{B}$ The emphasis on 'when' in this formulation is particularly important and contains within it a tacit time-dependence that has not been explored in full depth. The only new element in the Afshar experiment is the presence of the wire grid. As in Fig. 1, let the photon beams converge at a spatial location $\sigma_{1}$, where they pass through the wire grid. At location $\sigma_{2}$ let the photon beams hit the detectors, after which one is able to deduce both the existence of the interference pattern and the path information for the photons. We argue below that these facts do not constitute a violation of the Bohr complementarity principle as claimed by Afshar $12 \mid 3$. Further, the wire grid is superfluous in the sense that it is not required to deduce the existence of the interference pattern; the complete path information is sufficient for this purpose.

Consider a photon that passes through the wire grid at time $t_{1}$ and then reaches a detector at time $t_{2}$. Afshar's argument is essentially that a large number of such photons that reach the detectors at time $t_{2}$ must have formed an interference pattern at time $t_{1}$, because of the negligibly small number of photons that impinged on the wire grid at time $t_{1}$ (as compared to the number that reached the detectors). It is crucial to note that Afshar is able to argue for the existence of the interference pattern for this large number of photons only after they have reached the detectors, at which point of time complete path information is also available for each of these photons. If these photon paths are superposed on to an imaginary screen at location $\sigma_{1}$, a complete reconstruction of the interference pattern is theoretically possible, without any need for the wire grid. Therefore if one accepts Afshar's argument that the Bohr complementarity principle is violated in his experiment, one must also accept the same conclusion from a classical version of his experiment, in which the wire grid is not present. In fact the analysis of the path information would provide a complete reconstruction of the interference pattern, whose existence is only nonconstructively deduced via the wire grid. Hence the wire grid is not only logically superfluous in Afshar's argument, but it also provides a less satisfactory and controversial method for deducing the existence of an interference pattern. It is extremely important to note that Afshar also accepts the validity of the path information for a photon in his experiment, post its detection at time $t_{2}$. It follows that Afshar has also essentially analyzed particle-like photons, each of which he accepts as having passed through only one of the slits, to arrive at his conclusion that an interference pattern existed at a previous time $t_{1}$ for these photons.

We may conclude that Afshar's method of deducing (or as he claims, measuring) the existence of an interference pattern does not provide any evidence whatsoever of wave-like properties for a photon. Likewise, analysis of the path information in a classical version of Afshar's experiment (without the wire grid) would also provide a reconstruction of the interference pattern without providing any evidence whatsoever of wave-like properties for a photon. In contrast, the classical method

${ }^{b}$ The author is grateful to an anonymous referee for suggesting this formulation and the analogy of the quantum eraser for the Afshar experiment. 
for measuring an interference pattern, via a destruction of the photons by a screen located at $\sigma_{1}$, would eliminate any possibility of deducing path information for these photons. It is the non-availability of the path information at time $t_{1}$ that establishes the wave nature of the photon (via the superposed state $S$ ), whose logical consequence is the presence of an interference pattern. It is extremely important to note that the converse implication does not hold: the presence of an interference pattern does not logically imply that that the photons are in a superposed state. Hence the co-existence of the interference pattern and the path information, as deduced at time $t_{2}$, is not a contradiction as claimed by Afshar. Post the time $t_{2}$, the correct interpretation of the Afshar experiment (or a classical version without the wire grid) is that particle-like photons have a non-classical probability distribution, resulting in an interference pattern. Here one must understand the time dependence involved and give up any realist notions of the superposed state $S$, or the wave nature of the photon.

The state $S$ should be thought of as merely a formalism by which we may deduce probability distributions, which are confirmed by the existence of the interference pattern. The Copenhagen interpretation championed by Bohr has always been nonrealist in this sense. As we will see in the ensuing sections, in the (non-realist) NAFL interpretation, the state $S$ of the photons has the precise logical meaning that path information is not available; it does not mean that a single photon 'really' took both paths, which is a logical impossibility for a particle. Secondly, the retroactive assertion of the path information at time $t_{2}$ is a logical truth that only applies for times $t \geq t_{2}$; it does not mean that the path information was always available. Again, one needs a non-classical, temporal logic like NAFL to formulate this time-dependence, which, however, was always tacitly implied by the Copenhagen interpretation. Seen in this light, we know the following facts about the modified Afshar experiment. Firstly, at time $t_{1}$, path information is not available for the photons that are passing through the wire grid (and which will impinge on the detectors at time $t_{2}$ ), and hence they are in the superposed state $S$ at that instant $\left(t_{1}\right)$. The interference pattern is a logical consequence of this state. Secondly, at time $t_{2}$, path information is retroactively available for the photons that impinged on the detectors at that instant; however, this retroactive assertion itself only applies for times $t \geq t_{2}$. The inferred presence of the interference pattern does not logically imply that the photons are in a superposed state $S$; it is this fact which permits a retroactive assertion of the path information post the time $t_{2}$.

A contradiction would ensue from the above two facts if one insists on ascribing reality for the superposed state $S$ and also insists that the retroactive assertion of the path information was always applicable (as would be the case in classical logic). In the first case one would have the contradiction that the photons 'really' took both paths and the photons took only one of the available paths; in the second case the contradiction would be that path information is both available and not available

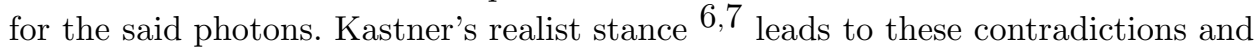
hence forces her to reject the retroactive assertion of the path information even 
in the case when the wire grid is not present (as noted in Sec. 11). This stance contradicts well-accepted results in physics and fails to explain how a photon can 'really' take both paths and yet exhibit particle-like behaviour at the detectors (decoherence).

The Bohr complementarity principle is formulated in the NAFL interpretation as a logical principle (see Sec. 4.2). Namely, that at any given time, a photon can either be in a superposed state, in which case the law of noncontradiction fails, or in a classical particle-like state, when the law of noncontradiction applies; at any given time, it is not logically possible for the photon to be in both of these states. This formulation reduces to that noted earlier in terms of which-way information and interference information. The Englert-Greenberger duality relation 4|5, when properly interpreted, is also upheld in the Afshar experiment, contrary to Afshar's claims $1 / 2 \mid 3$. This relation may be expressed in the form

$$
D^{2}+V^{2} \leq 1
$$

Here $V$ is the visibility of the interference fringes and $D$ is the distinguishability of the photon paths. Afshar claims that in his experiment, both $D$ and $V$ are close to 1, and hence Eq. (11) is violated. However, if the time dependence of the parameters $D$ and $V$ is taken into account, one may conclude that Eq. (11) is indeed upheld in the Afshar experiment. At time $t_{1}$, when the photons pass through the wire grid, there is no path information and hence $D=0$ for these photons; further, the photons are in a superposed state at this time with the interference fringes present, and hence $V=1$. Indeed, this is certainly true for the photons that impinge on the wire grid. Post the time $t_{2}$, path information is available for the photons that impinge on the detectors, and hence we take $D=1$ for these photons. As noted earlier, we may infer that these photons were part of an interference pattern at time $t_{1}$, regardless of whether the wire grid is present. Despite this inference, one concludes that $V=0$ for time $t \geq t_{2}$, because the presence of the interference pattern does not imply that the photons are in a superposed state and so the path information stands for these times. And when path information is available, the interference pattern is no longer a logical consequence of any wave-like properties of the photon, which is why we take $V=0$ at these times. When $D$ and $V$ are interpreted in this manner, it is impossible for Eq. (11) to be violated in the Afshar experiment (with or without the wire grid) because it expresses precisely the Bohr complementarity principle.

Finally, consider the analogy of the Schrödinger cat experiment (see Sec. 3.3). At time $t_{1}$, let us say that the cat is in the box and is in a superposed state of 'alive and dead'. What this state means in the NAFL interpretation is that no information is available at time $t_{1}$ as to the cat's classical state ('alive' or 'dead'). Subsequently, at time $t_{2}$, when the box is opened, suppose the cat is found in the 'alive' state. One may retroactively infer at time $t_{2}$ that the cat was alive at time $t_{1}$. This retroactive inference only applies for times $t \geq t_{2}$ and does not contradict the fact that at time $t_{1}$, the cat was in a superposed state (to which no 'reality' can be ascribed). Again the complementarity principle is upheld in both the NAFL and 
the Copenhagen interpretations because at any given time, the cat is in only one state, despite the retroactive inference. Whereas Kastner's interpretation 617 would amount to barring the retroactive inference that the cat was in the 'alive' state at time $t_{1}$ because it 'really' was in the superposed state of 'alive and dead' at that time. Any such 'reality' is obviously aphysical and inexplicable, and therefore untenable in our view.

\section{The NAFL interpretation of quantum superposition}

At the outset, we hasten to note that the NAFL interpretation is still nascent and incomplete in the sense that a lot of work remains to be done in demonstrating how real analysis can be done in NAFL 18 , and ultimately, how all of quantum mechanics can be formalized in this logic. What has been accomplished at this stage is a completely new and logical interpretation of some of the "weird" phenomena of quantum mechanics, in particular, superposition and entanglement 15/16]17. In this section, we will confine ourselves to a brief exposition of the NAFL interpretation of quantum superposition, and refer the reader to the original references for further details. The reader who is already familiar with these details may skip to Sec.4 Our purpose herein is to provide just enough information on NAFL so as to enable an appreciation of the delicate and subtle logical issues involved in the interpretation of the Bohr complementarity principle in Afshar's experiment, which is discussed in Sec. 4 .

The language, well-formed formulae and rules of inference of NAFL theories 15 are formulated in exactly the same manner as in classical first-order predicate logic with equality (FOPL), where we shall assume, for convenience, that natural deduction is used; however, there are key differences and restrictions imposed by the requirements of the Main Postulate of NAFL, which is explained in this section. In NAFL, truths for formal propositions can exist only with respect to axiomatic theories. There are no absolute truths in just the language of a NAFL theory, unlike classical/intuitionistic/constructive logics. There do exist absolute (metamathematical, Platonic) truths in NAFL, but these are truths about axiomatic theories and their models. As in FOPL, a NAFL theory $\mathrm{T}$ is defined to be consistent if and only if $\mathrm{T}$ has a model, and a proposition $P$ is undecidable in $\mathrm{T}$ if and only if neither $P$ nor its negation $\neg P$ is provable in $\mathrm{T}$.

\subsection{The Main Postulate of NAFL}

If a proposition $P$ is provable/refutable in a consistent NAFL theory $\mathrm{T}$, then $P$ is true/false with respect to $\mathrm{T}$ (henceforth abbreviated as 'true/false in $\mathrm{T}$ '); i.e., a model for T will assign $P$ to be true/false. If $P$ is undecidable in a consistent NAFL theory $\mathrm{T}$, then the Main Postulate 16 provides the appropriate truth definition as follows: $P$ is true/false in $\mathrm{T}$ if and only if $P$ is provable/refutable in an interpretation $\mathrm{T}^{*}$ of $\mathrm{T}$. Here $\mathrm{T}^{*}$ is an axiomatic NAFL theory that, like $\mathrm{T}$, temporarily resides in the human mind and acts as a 'truth-maker' for (a model of) $\mathrm{T}$. The theorems of $\mathrm{T}^{*}$ 
are precisely those propositions that are assigned 'true' in the NAFL model of T, which, unlike its classical counterpart, is not 'pre-existing' and is instantaneously generated by $\mathrm{T}^{*}$. It follows that $\mathrm{T}^{*}$ must necessarily prove all the theorems of $\mathrm{T}$. Note that for a given consistent theory $\mathrm{T}, \mathrm{T}^{*}$ could vary in time according to the free will of the human mind that interprets $\mathrm{T}$; for example, $\mathrm{T}^{*}$ could be $\mathrm{T}+P$ or $\mathrm{T}+\neg P$ or just $\mathrm{T}$ itself at different times for a given human mind, or in the context of quantum mechanics, for a given observer. Further, $\mathrm{T}^{*}$ could vary from one observer to another at any given time; each observer determines $\mathrm{T}^{*}$ by his or her own free will. The essence of the Main Postulate is that $P$ is true/false in $\mathrm{T}$ if and only if it has been axiomatically declared as true/false by virtue of its provability/refutability in $\mathrm{T}^{*}$. In the absence of any such axiomatic declarations, i.e., if $P$ is undecidable in $\mathrm{T}^{*}\left(\right.$ e.g. take $\left.\mathrm{T}^{*}=\mathrm{T}\right)$, then $P$ is 'neither true nor false' in $\mathrm{T}$ and Proposition 1 shows that consistency of $\mathrm{T}$ requires the laws of the excluded middle and noncontradiction to fail in a nonclassical model for $\mathrm{T}$ in which $P \& \neg P$ is the case.

Proposition 1. Let $P$ be undecidable in a consistent NAFL theory T. Then $P \vee \neg P$ and $\neg(P \& \neg P)$ are not theorems of $\mathrm{T}$. There must exist a nonclassical model $\mathcal{M}$ for $\mathrm{T}$ in which $P \& \neg P$ is the case.

For a proof of Proposition 1, see Ref. 15] or Appendix A of Ref. 17, this proof also seriously questions the logical/philosophical basis for the law of noncontradiction in both classical and intuitionistic logics. The interpretation of $P \& \neg P$ in the nonclassical model will be explained in Sec. 3.2. Proposition 1 is a metatheorem, i.e., it is a theorem about axiomatic theories. The concepts in Proposition 1 namely, consistency, undecidability (or provability) and the existence of a nonclassical model for a theory and hence, quantum superposition and entanglement, are strictly metamathematical (i.e., pertaining to semantics or model theory) and not formalizable in the syntax of NAFL theories. A NAFL theory T is either consistent or inconsistent, and a proposition $P$ is either provable or refutable or undecidable in $\mathrm{T}$, i.e., the law of the excluded middle applies to these metamathematical truths. Note that the existence of a nonclassical model does not make $\mathrm{T}$ inconsistent or even paraconsistent in the conventional sense, because $\mathrm{T}$ does not prove $P \& \neg P$. However, one could assert that the model theory for $\mathrm{T}$ requires the framework of a paraconsistent logic, so that the nonclassical models can be analyzed. NAFL is the only logic that correctly embodies the philosophy of formalism 16; NAFL truths for formal propositions are axiomatic, mental constructs with strictly no Platonic world required.

\subsection{Quantum superposition justified in NAFL}

The nonclassical model $\mathcal{M}$ of Proposition 1 is a superposition of two or more classical models for $\mathrm{T}$, in at least one of which $P$ is true and $\neg P$ in another. Here '(non-)classical' is used strictly with respect to the status of $P$. In $\mathcal{M}$, ' $P$ ' (' $\neg P$ ') denotes that ' $\neg P$ ' (' $P$ ') is not provable in $\mathrm{T}^{*}$, or in other words, $\mathcal{M}$ expresses that 
neither $P$ nor $\neg P$ has been axiomatically declared as (classically) true with respect to T; thus $P, \neg P$, and hence $P \& \neg P$, are indeed (nonclassically) true in our world, according to their interpretation in $\mathcal{M}$. Note also that $P$ and $\neg P$ are classically 'neither true nor false' in $\mathcal{M}$, where 'true' and 'false' have the meanings given in the Main Postulate. The quantum superposition principle is justified by identifying 'axiomatic declarations' of truth/falsity of $P$ in $\mathrm{T}$ (via its provability/refutability in $\mathrm{T}^{*}$ as defined in the Main Postulate) with 'measurement' in the real world. NAFL is more in tune with the Copenhagen interpretation of quantum mechanics than the many-worlds interpretation (MWI). Nevertheless, the information content in $\mathcal{M}$ is that of two or more classical models (or 'worlds'), and MWI is at least partially vindicated in this sense.

\subsection{Example: Schrödinger's cat}

Consider the situation wherein the cat is put into the box at time $t=t_{0}$ and has a probability 0.5 of being in the 'alive' state at $t=t_{2}$, when a 'measurement' is made of its state. Let $P$ be the proposition that 'The cat is alive', with $\neg P$ denoting 'The cat is dead'; obviously, $P$ is undecidable in a suitable formalization QM of quantum mechanics, which may be taken to include definitions describing this experiment. For $t_{0}<t<t_{2}$, the observer makes no measurements, and in tune with the identification noted in Sec. 3.2 makes no axiomatic declarations regarding $P$ in the interpretation $\mathrm{QM}^{*}$ (say, let $\mathrm{QM}^{*}=\mathrm{QM}$ for this time period). In the resulting nonclassical model $\mathcal{M}$ of $\mathrm{QM}$, the superposed state $P \& \neg P$ is the case; this means that the cat has not been declared (measured) to be either alive or dead, which is certainly true in the real world. At $t=t_{2}$, if $P(\neg P)$ is observed, then the observer takes, say, $\mathrm{QM}^{*}=\mathrm{QM}+V(\neg V)$, where $V(\neg V)$ is defined as 'The cat is alive (dead) at $t=t_{2}$ '; note that $\mathrm{QM}^{*}$ will prove $P$ (or $\neg P$ ) at $t=t_{2}$, i.e., when the observer measures the cat to be alive (dead) in the real world, he makes the appropriate axiomatic declarations in his mind, thus setting up QM* as defined. It should be emphasized that a NAFL theory only 'sees' the observer's axiomatic declarations and does not care whether the real world exists. The observer sees the real world and the proposed identification of his measurements with his axiomatic declarations is only an informal convention that is outside the purview of NAFL. The observer could also use his free will to make his axiomatic declarations irrespective of (and possibly in contradiction to) what he measures in the real world; of course, if $P$ is not about the real world, then he has no other choice. NAFL correctly handles the temporal nature of truth via the time-dependence of $\mathrm{QM}^{*}$. If $P$ is observed (and axiomatically asserted via $\mathrm{QM}^{*}=\mathrm{QM}+V$ ) at $t=t_{2}$, then the proposition $U$ that "The cat was alive for $t_{0}<t<t_{2}$ " can be formalized for $t \geq t_{2}$ and proven in the NAFL theory $\mathrm{QM}^{*}=\mathrm{QM}+V ; U$ does not conflict temporally or in meaning with the superposed state $P \& \neg P$, which applies for $t_{0}<t<t_{2}$. We

will return to the Schrödinger cat example in Sec. 5 , in order to further elaborate upon the validity of this retroactive assertion of $U$. 


\subsection{Theory syntax and proof syntax}

A NAFL theory T requires two levels of syntax, namely the 'theory syntax' and the 'proof syntax'. The theory syntax consists of precisely those propositions that are legitimate, i.e., whose truth in T satisfies the Main Postulate; obviously, the axioms and theorems of $\mathrm{T}$ are required to be in the theory syntax. Further, one can only add as axioms to $\mathrm{T}$ those propositions that are in its theory syntax. In particular, neither $P \& \neg P$ nor its negation $P \vee \neg P$ is in the theory syntax when $P$ is undecidable in T. The proof syntax, however, is classical because NAFL has the same rules of inference as FOPL; thus $\neg(P \& \neg P)$ is a valid deduction in the proof syntax and may be used to prove theorems of T. For example, if one is able to deduce $A \Rightarrow P \& \neg P$ in the proof syntax of $\mathrm{T}$ where $P$ is undecidable in $\mathrm{T}$ and $A$ is in the theory syntax, then one has proved $\neg A$ in $\mathrm{T}$ despite the fact that $\neg(P \& \neg P)$ is not a theorem (in fact not even a legitimate proposition) of $\mathrm{T}$. This is justified as follows: $\neg(P \& \neg P)$ may be needed to prove theorems of $\mathrm{T}$, but it does not follow in NAFL that the theorems of T imply $\neg(P \& \neg P)$ if $P$ is undecidable in T. Let $A$ and $B$ be undecidable propositions in the theory syntax of T. Then $A \Rightarrow B$ (equivalently, $\neg A \vee B$ ) is in the theory syntax of $\mathrm{T}$ if and only if $A \Rightarrow B$ is not (classically) deducible in the proof syntax of $\mathrm{T}$. It is easy to check that if $A \Rightarrow B$ is deducible in the proof syntax of $\mathrm{T}$, then its (illegal) presence in the theory syntax would force it to be a theorem of $\mathrm{T}$, which is not permitted by the Main Postulate. For, in a nonclassical model $\mathcal{M}$ for $\mathrm{T}$ in which both $A$ and $B$ are in the superposed state, $A \& \neg B$ must be nonclassically true, but theoremhood of $A \Rightarrow B$ will prevent the existence of $\mathcal{M}$. If one replaces $B$ by $A$ in this result, one obtains the previous conclusion that $\neg(A \& \neg A)$ is not in the theory syntax. For example, take $T_{0}$ to be the null set of axioms. Then nothing is provable in $\mathrm{T}_{0}$, i.e., every legitimate proposition of $\mathrm{T}_{0}$ is undecidable in $\mathrm{T}_{0}$. In particular, the proposition $(A \&(A \Rightarrow B)) \Rightarrow B$, which is deducible in the proof syntax of $\mathrm{T}_{0}$ (via the modus ponens inference rule), is not in the theory syntax; however, if $A \Rightarrow B$ is not deducible in the proof syntax of $\mathrm{T}_{0}$, then it is in the theory syntax. Note also that $\neg \neg A \Leftrightarrow A$ is not in the theory syntax of $\mathrm{T}_{0}$; nevertheless, the 'equivalence' between $\neg \neg A$ and $A$ holds in the sense that one can be replaced by the other in every model of $\mathrm{T}_{0}$, and hence in all NAFL theories. Indeed, in a nonclassical model for $\mathrm{T}_{0}$, this equivalence holds in a nonclassical sense and must be expressed by a different notation 16 .

\section{Bohr Complementarity and the NAFL interpretation of Afshar's experiment}

The Bohr complementarity principle easily follows from the NAFL interpretation of quantum superposition discussed in Sec. 3. As applied to Afshar's experiment, the relevant definition is as follows.

Definition 1 (Bohr complementarity principle (BCP)). The particle and 
wave nature of the photon cannot be simultaneously demonstrated to hold at any given spatial location and time within a given experiment.

Note carefully the location of the word "simultaneously" in this definition; if we were to change BCP to "... cannot be demonstrated to hold simultaneously at any given spatial location and time within a given experiment", then such a definition would arguably fail even in NAFL. The ability of NAFL to handle the temporal nature of mathematical truth and the distinctions NAFL makes between syntax and semantics are demonstrated to be very important for a correct logical explanation of the results in Afshar's experiment.

\subsection{Quantum superposition in Afshar's experiment}

Let QM be the NAFL theory formalizing quantum mechanics. We assume that definitions providing a detailed description of the single-photon version of Afshar's experiment 112]3 (which he has reportedly performed with the same results) have already been included in QM.

Definition 2. Let $P$ denote the proposition "The photon passed through (only) slit $\mathrm{U}$ at location $\sigma$ and time $t_{0}$ in Fig. 11"

Definition 3. Let the negation $\neg P$ of $P$ denote the proposition "The photon passed through (only) slit L at location $\sigma$ and time $t_{0}$ in Fig. 1."

Since $P$ and $\neg P$ are equally probable, it follows that $P$ is undecidable in QM. Here it is important to understand the NAFL concept of negation (see Sec. 2.2 of Ref. 16); in particular, mutually exclusive classical possibilities (e.g. in the real world) are negations of each other and $\neg \neg P$ is equivalent to $P$, just as in classical logic. But unlike classical logic, $P \vee \neg P$ is not a theorem of $\mathrm{QM}$, and unlike intuitionistic logic, $\neg(P \& \neg P)$ is also not a theorem of QM; as noted in Sec. 3.1 there must exist a nonclassical model $\mathcal{M}$ for $\mathrm{QM}$ in which $P \& \neg P$ is the case. The interpretation of $P \& \neg P$ in $\mathcal{M}$ is identical to that explained in Secs. 3.2 and 3.3 ' $P$ ' (resp. ' $\neg P$ ') of $P \& \neg P$ has the nonclassical meaning that ' $\neg P$ ' (resp. ' $P$ ') is not provable in the observer's interpretation $\mathrm{QM}^{*}$ of $\mathrm{QM}$. In keeping with the informal convention noted in Secs. 3.2 and 3.3. the observer agrees to keep his axiomatic assertions in tune with his measurements in the real world, so that the superposition $P \& \neg P$ in $\mathcal{M}$ also has an equivalent nonclassical meaning with ' $P$ ' (resp. ' $\neg P$ ') now denoting that "The observer has not measured the photon to pass through slit L (resp. slit U) at location $\sigma$ and time $t_{0}$ in Fig. 1," One can see that the NAFL interpretation of $P \& \neg P$ is meaningful in the real world because $\neg P$ is not really the negation of $P$ in $\mathcal{M}$; if the observer has not measured (axiomatically declared) that the photon passed through slit L, it does not follow that he has measured (axiomatically declared) that the photon passed through slit U. Consistency demands that the observer should never be able to prove $P \& \neg P$ in any NAFL theory, in particular, QM or $\mathrm{QM}^{*}$; for such a proof would imply the contradiction: "The photon 
really passed through both slits". The only reality that exists, as far as the observer is concerned, is that he has not measured or axiomatically asserted either $P$ or $\neg P$ in the real world. This same reality is accurately modeled in the nonclassical model $\mathcal{M}$ where, somewhat paradoxically, $P, \neg P$ and $P \& \neg P$ all hold, but with the nonclassical interpretations noted above; the existence of such a nonclassical model for QM is a requirement of consistency in NAFL.

NAFL requires that both $P \& \neg P$ and its negation $\neg(P \& \neg P$ ) (or equivalently, $P \vee \neg P)$ are not legitimate propositions in the theory syntax of QM; see Sec. 3.4 What this means is that formally, the status of the photon in the theory syntax of $\mathrm{QM}$ is indeterminate; it cannot be proven to be either a particle (in which case $P \vee \neg P$ should be a theorem) or a wave (in which case $P \& \neg P$ should be a theorem). Consequently, both classical and nonclassical models, with respect to the proposition $P$, exist for QM. Note that the NAFL semantics for the nonclassical model $\mathcal{M}$ of QM does not take a stand either on the status of the photon as a particle or non-particle. Nevertheless, the NAFL theory QM tacitly supports (but not requires) the 'reality' of the particle nature of the photon in a metalogical sense, i.e., outside of both theory syntax and semantics. This will be fully explained in Sec. 4.2 Here we observe that the proof syntax of QM requires the deduction of $P \vee \neg P$; see Sec. 3.4, where it is noted that the rules of inference of NAFL theories, which determine the proof syntax, must be classical. It is possible (but not necessary) to interpret this deduction as a metalogical assertion of the particle nature of the photon in the sense that the photon 'really' had to pass through one and only one of the two slits $\mathrm{U}$ and $\mathrm{L}$ at time $t_{0}$ in Fig. 1, even though, at that instant, the observer did not have a proof that either of these paths was traversed. It is this lack of knowledge at time $t_{0}$ that NAFL semantics expresses as a requirement of logical consistency, via the nonclassical model $\mathcal{M}$, rather than any perceived reality for the particle nature of the photon. But such a perceived reality became inevitable the moment Definition 3 was formulated in QM as the negation of $P$. Here it should be kept in mind that the 'photon' referred to is that which must necessarily pass through at least one of the slits. The coin toss experiment, considered in Sec. 5. will further illustrate the metalogical 'reality' of $P \vee \neg P$ in NAFL.

\subsection{Bohr Complementarity in Afshar's experiment}

Consider again the single-photon version of Afshar's experiment in Fig. 11, with $P$, $\neg P$ and the theory $\mathrm{QM}$ as defined in Sec. 4.1. At time $t=t_{0}$ a photon passes through the slit(s); at $t=t_{1}>t_{0}$, the said photon passes the wire grid in the "interference plane" at location $\sigma_{1}$, and subsequently passes through the converging lens; at $t=t_{2}>t_{1}$, the photon ends up at one of the locations marked $\mathrm{U}^{\prime}$ or $\mathrm{L}^{\prime}$ (say, $\mathrm{U}^{\prime}$, for the sake of definiteness). Note that in the single-photon version of Afshar's experiment, the restriction is that only one photon at a time can pass through the slit(s), although several photons actually end up at $\mathrm{U}^{\prime}$ and $\mathrm{L}^{\prime}$. 
Definition 4. Take the proposition $Q$ to denote that "The photon reaches the image $\mathrm{U}^{\prime}$ of slit $\mathrm{U}$ at location $\sigma_{2}$ and time $t_{2}$ in Fig. 1]'

Definition 5. Let the proposition $R$ denote that "The images $\mathrm{L}^{\prime}$ and $\mathrm{U}^{\prime}$ of the slits $\mathrm{L}$ and $\mathrm{U}$ respectively are undistorted and unchanged in intensity when the wire grid is inserted at the calculated minima of the expected interference pattern at location $\sigma_{1}$ in Fig. 11'.

Consider the following contentious inferences that are inherent in the controversy over Afshar's experiment.

Inference 1. The theory $\mathrm{QM}+Q$ proves $P$.

This, of course, implies the particle nature of the photon.

Inference 2. The theory $\mathrm{QM}+R$ proves $P \& \neg P$.

In words, Inference 2 means "The photon passed through both slits $\mathrm{U}$ and $\mathrm{L}$ at location $\sigma$ and time $t_{0}$ in Fig. 11', which in turn implies the wave nature of the photon. Since $Q$ and $R$ are true, in the sense that they are observed, one may conclude that if Inferences 1 and 2 are granted, the resulting 'true' (but inconsistent) theory $\mathrm{QM}+Q+R$ violates BCP; see Definition 11. Kastner 617 seems to permit Inference 1, but disputes that it 'really' establishes 'which-way' information for the photon; she argues that Cramer's Transactional Interpretation 19 supports her stand. Kastner concludes that only the wave nature of the photon is unambiguously exhibited at the slits in Afshar's experiment, via Inference 2, which she allows (although neither she nor Afshar interpret the wave nature of the photon as a proposition of the form $P \& \neg P$, as NAFL requires). In what follows, we will argue that the NAFL interpretation allows Inference1 but not Inference 2 this fact, when coupled with the temporal nature of mathematical truth in NAFL, means that Bohr complementarity survives. The claim that a single photon 'really' exhibits wave nature is questionable from the NAFL point of view and will be critically examined in Sec. 4.3. However, we emphasize at the outset that the wave nature of light could well follow unambiguously in a new, as yet unknown, version of QM formalized in NAFL. The present argument applies only to our current understanding of QM and the nature of light.

The NAFL justification of BCP is as follows. For $t_{0} \leq t<t_{2}$, the observer has the theory $\mathrm{QM}$ in mind with the interpretation $\mathrm{QM}^{*}=\mathrm{QM}$. Hence by Proposition 1 (see Sec. 3.1),$P \& \neg P$ holds for the observer, in a nonclassical model $\mathcal{M}$ for QM. At $t=t_{2}$, upon measuring $Q$, the observer switches to the interpretation $\mathrm{QM}^{*}=\mathrm{QM}+Q$ and concludes $P$ in a classical model for $\mathrm{QM}$, via a proof in $\mathrm{QM}^{*}$ (Inference 1). Note that $P$ only applies retroactively, for times $t \geq t_{2}$; therefore it does not temporally conflict with the superposition $P \& \neg P$, which applied for $t_{0} \leq t<t_{2}$. The second observation here is that the theory QM does not prove $P \& \neg P$; as noted earlier, $P \& \neg P$ is not even a legitimate proposition in the theory syntax of QM. Such a proof of $P \& \neg P$ would make QM inconsistent in NAFL, which 
is not a conventional paraconsistent logic, as noted in Sec. 3.1. In fact, the Main Postulate of NAFL and consequently, the existence of $\mathcal{M}$, are strictly metamathematical results, i.e., pertaining to semantics or model theory; these concepts are not formalizable in either the theory syntax or proof syntax of QM. The theory $\mathrm{QM}^{*}$ can therefore prove $P$ without loss of consistency. Since the deduction of $P$ and the meta-deduction of the superposition $P \& \neg P$ were made in non-overlapping time intervals, and, in particular, were not made simultaneously, BCP survives in NAFL.

One might object that there is something unsatisfactory about this state of affairs; if we interpret $P$ and $P \& \neg P$ as affirming the particle and the wave natures of the photon respectively, they seem to be contradictory even if asserted at nonoverlapping time intervals, since, after all, they both apply to the same photon at the same spatial location and time; this is in fact the essence of Afshar's argument 1 . The answer to this objection is that in the nonclassical model $\mathcal{M}, P \& \neg P$ only means that the observer has not axiomatically asserted $P$ via a proof in $\mathrm{QM}^{*}$ (or measured $P$ in the real world) and he has not axiomatically asserted $\neg P$ via a proof in $\mathrm{QM}^{*}$ (or measured $\neg P$ in the real world); as noted in Sec.4.1 $P \& \neg P$ does not imply that the photon 'really' exhibited wave nature and passed through both slits. But the retroactive assertion of $P$ (via a proof in $\mathrm{QM}^{*}$ ), valid for $t \geq t_{2}$, can be taken to have the metalogical (see Sec. 4.1) meaning that the photon 'really' passed through only slit $\mathrm{U}$ at time $t_{0}$, and therefore does not conflict in meaning with the meta-deduction of $P \& \neg P$ made in $\mathcal{M}$ during the time interval $t_{0} \leq t<t_{2}$; both are indeed 'true' when appropriately interpreted. To summarize, for $t_{0} \leq t<t_{2}$, NAFL semantics only recognizes the metamathematical truth of $P \& \neg P$ in $\mathcal{M}$. For $t \geq t_{2}$, NAFL semantics asserts the retroactive truth of $P$ via a classical model for QM; this truth may be taken to hold metalogically for $t_{0} \leq t<t_{2}$, i.e., outside of NAFL syntax and semantics. The temporal nature of NAFL truth plays a vital role in removing the mystery associated with wave-particle duality. In classical logic, unlike NAFL, the retroactive assertion of $P$ at $t=t_{2}$ would necessarily mean that $P$ was always true in the semantics of $\mathrm{QM}$, including at $t=t_{0}$. Therefore in the framework of classical logic, such a retroactive assertion of $P$ will be problematic and will clash with the quantum superposition state that actually held in the semantics of QM at $t=t_{0}$.

At this stage the reader will have the following obvious question; does not $R$ (Definition 5) prove the existence of the interference pattern at location $\sigma_{1}$ of Fig. 1 and consequently, the reality of the wave nature of the photon when it enters the slits $\mathrm{U}$ and L? In the NAFL interpretation, the answer to the second part of this question is in the negative; in Sec. 4.3 we will argue that Inference 2 is not permitted on logical grounds and therefore the observation $R$ is irrelevant to the above-noted retroactive conclusion of $P$ by the observer. A second question that arises is whether there are logical grounds for banning Inference 1 as well. Afshar 1 has asserted that Inference 1 follows from standard optics and has subsequently pointed out elsewhere that moving one of the slits U or L in Fig. 1 causes the cor- 
responding image $\mathrm{U}^{\prime}$ or $\mathrm{L}^{\prime}$ to co-move with the slit. Here we do not pass judgement on the experimental validity of Afshar's claims. We only wish to point out that on purely logical grounds, NAFL does permit the conclusion of Inference 1. namely, the retroactive assertion of $P$; for reasons mentioned in Sec. 4.1 and in this subsection, NAFL does not object to the metalogical existence of the photon as a particle. Thus one could also take the interpretation at $t=t_{2}$ as $\mathrm{QM}^{*}=\mathrm{QM}+P$ (instead of $\mathrm{QM}^{*}=\mathrm{QM}+Q$ as noted above), if it turns out that there is indeed something wrong with Inference 1.

We note that although the NAFL interpretation does permit the retroactive assertion of $P$, via Inference 1 or otherwise, there is no obligation on the part of the observer to make such an assertion (or measurement). The observer can choose to live with just the metamathematical conclusion that both classical and nonclassical models for QM exist; he could choose to remain agnostic (i.e., take no stand) on the metalogical status of the photon as a particle or non-particle in the nonclassical models and scrupulously avoid making any retroactive assertions/measurements. Consequently, to facilitate such an agnostic attitude, Inference 1 must either be disallowed or if permitted, must be weakened so as not to imply any 'reality' for the path of the photon at time $t_{0}$. At time $t_{2}$, the photon behaves as though it originated from slit U (via the weakened Inference 1), but need not have 'really' done so, from the agnostic's point of view. Kastner $6 / 7$ seems to prefer this latter approach to Inference 1: however, rather than remain agnostic, she asserts that the photon passed through both slits, as a wave. In Sec. 4.4, we criticize the limiting of Inference 1 as noted above, as well as Kastner's reasons for doing so. Cramer's Transactional Interpretation 19 is also criticized from the NAFL point of view in Sec. 4.5. An anti-realist approach is also possible in NAFL. Anti-realism is stronger than agnosticism, in the sense that it requires the observer to deny any reality for the state of the photon as particle or non-particle in the nonclassical model $\mathcal{M}$. In other words, no such reality exists in the absence of an axiomatic declaration, which, by informal convention, the observer associates with 'measurement' in the real world. The anti-realist approach would presumably require the banning of Inference 1 and other retroactive assertions of reality. Of course, Inference 2 is already illegal in NAFL, as will be explained in Sec. 4.3 .

In summary, there are three approaches possible in NAFL regarding the status of the photon during $t_{0} \leq t<t_{2}$, when $\mathcal{M}$ is in force. These are, a metalogical reality for the particle state, agnosticism and anti-realism. The author believes that the first option is the most satisfactory from both philosophical and logical points of view, given Definition 3. With such a choice of negation in NAFL, has the observer denied any possibility that the photon can 'really' pass through both slits, thereby affirming its metalogical particle status? This is a tricky issue; the author believes that the answer is in the positive, i.e., the observer has predetermined the particle nature of the photon via Definition 3 One may attempt to justify the above choice of negation by arguing that $P$ and $\neg P$ cannot be 'measured' together, 
although $P \& \neg P$ could be 'really' true in the real world (this would provide another reason for banning Inference 2, for otherwise the proposition $R$ in Definition 5 surely amounts to a 'measurement' of $P \& \neg P)$. However, such an attempt at justification fails, for the following reason. NAFL truth for undecidable propositions of a theory is purely axiomatic in nature, by the Main Postulate (see Sec. 3.1); the concept of 'measurement' cannot be formalized in the theory syntax of NAFL theories 15 . The informal convention of associating 'axiomatic declaration' with 'measurement' in the real world can, in principle, be broken in NAFL; see Sec. 3.3 as well as the final paragraph of Sec. 4.3. If the photon can 'really' (or classically) pass through both slits, there ought to be nothing to stop us from axiomatically declaring, i.e., inferring via a proof in $\mathrm{QM}^{*}$, that 'The photon passed through both slits at $t=t_{0}$ ', even if measurement of this event is impossible. For such a proposition to be legal in the theory syntax of QM, NAFL would require the negation of $P$ to be modified such that it includes the case of the photon passing through both slits in disjunction with the case noted in Definition 3. However, as a consequence, BCP will fail by the Main Postulate of NAFL. For if both the particle and wave natures of the photon are classically possible phenomena, then the formal undecidability in QM of whether the photon exists as a particle or a wave would demand (via Proposition 1) that there must exist a nonclassical model $\mathcal{N}$ for QM in which the superposed state of the photon as a particle and a wave must hold. In $\mathcal{N}$, the photon will be neither a particle nor a wave, in violation of BCP. As explained in Sec. 4.3, we do not believe that this is the correct approach.

Reverting to the negation in Definition 3, the "wave nature" of the photon is a proposition of the form $P \& \neg P$. As noted earlier, neither $P \& \neg P$ nor its negation $P \vee \neg P$ (which symbolizes the particle nature of the photon) is a legitimate proposition in the theory syntax of QM. Consequently, BCP survives, because the Main Postulate and Proposition 1 1apply only to formal propositions that are in the theory syntax of NAFL theories; the nonclassical model $\mathcal{N}$ noted above need not (and does not) exist. Here we have put "wave nature" in quotes because $P \& \neg P$ in the nonclassical model $\mathcal{M}$ of QM does not imply that the photon is 'really' a wave, as was argued above and in Sec. 4.1. One can also state BCP, in the context of the NAFL interpretation of Afshar's experiment, as follows:

At any given time, the observer, via the interpretation $\mathrm{QM}^{*}$, can generate either a classical or a nonclassical model (but not both) for QM, with respect to the proposition $P$; in the classical model, $P \vee \neg P$ (and either $P$ or $\neg P$ ) holds, and the photon is a particle; in the nonclassical model, $P \& \neg P$ holds and the photon may be loosely termed as a 'wave', although its true status in the real world is left ambiguous.

One can see that the above formulation of BCP is not violated in the NAFL interpretation of Afshar's experiment. The NAFL interpretation also neatly solves the problem of the mysterious "instantaneous collapse of the wavefunction", which 
would arise if and only if one insists that the photon 'really' exhibits wave nature. Indeed, when $Q$ is measured at time $t_{2}$ in Fig. 1 all that happens is that the observer switches his interpretation $\mathrm{QM}^{*}$ of QM as noted previously. This amounts to a switch from a state of ignorance regarding the path of the photon to one of knowledge. Obviously, there is no implication here that the photon abruptly collapsed from a wave to a particle at $t=t_{2}$.

\subsection{Critique of Inference 2}

From the point of view of logic (and in particular, the NAFL interpretation), we wish to establish that the presence of an interference pattern, such as, that observed in Young's two-slit experiment, does not prove the 'reality' of the wave nature of a single photon.

Let us first consider the single-photon version of Young's two-slit experiment. Let the wire grid at location $\sigma_{1}$ in Fig. 1 1 be replaced by an electronic screen, capable of registering and storing the arrival of single photons. The photons reach the slits one at a time, with equal probability of passing through either slit. In the standard quantum formalism, a photon is assumed to take all available paths to any particular spot on the screen at which it ends up, i.e., the photon is assumed to pass through both slits and 'interfere with itself', in order to theoretically predict the interference fringes. Since these predictions agree with the experimental observations, the 'reality' of the wave nature of the photon is concluded. At the outset, let us grant that the interference fringes are indeed observed even in the single-photon case, even though doubts have been expressed in this regard when the rate of emission of photons is sufficiently small (Ref. 22, see the paragraph "If quanta are to be treated as real particles, self-interference must be ruled out. ... Nevertheless, this still means that the evidence in favour of self-interference is inconclusive."). Firstly, note that when a single photon is fired at the slits, it also ends up as a single, bright spot at a specific, unpredictable location on the screen; it does not exhibit an interference pattern on the screen. This fact conclusively establishes the grainy nature of the photon as it interacts with the screen. The interference pattern, which is observed to build up over time only after many photons have landed on the screen, has to be interpreted as reflecting a probability distribution, with the probability density function (PDF) proportional to some power of the intensity of the fringes. In particular, at the dark fringes of the screen, the photon has a vanishing PDF. Note that the PDF for the photon is normalized over the entire area of the $\sigma_{1}$ plane in which the screen is located.

Remark 1. A zero value of the probability density function (PDF) for the photon, at a point or a line located on a dark fringe (minimum) of the interference pattern on the screen in Young's two-slit experiment, does not imply a proof in the theory QM that a given photon cannot land at that point or line and be detected.

Remark 1 seems to follow even in the standard formalism for QM. Note that 
the probability (as opposed to the PDF) of the photon reaching any given point or line on the screen is exactly zero; but obviously this does not constitute a proof that the photon cannot be detected at that point or line. Points/lines on which the PDF vanishes, such as, the dark fringes in the two-slit experiment, are not special in this regard. An arbitrarily small area around such a point or line will still have a non-zero probability of recording a photon, as long as the PDF does not vanish identically in the entire area. So there does not appear to be any basis for excluding the possibility that a photon can land at the minima of the fringes, although one could expect that such an eventuality is unlikely in the real world. But one should not confuse 'statistical expectation' with 'proof'. To be sure, the observed intensity at the dark fringes is zero, and zero intensity means zero photon count rate. But remember that we are asking if a single, given photon can land at the dark fringes; 'count rate' and 'intensity' and even 'probability' are not really well-defined for this process. For example, a very large number of photons $(N)$ could be fired at the slits and $N-1$ of these could conform precisely to the expected probability distribution; if the remaining photon ends up at a dark fringe, that does not constitute a violation of any law of QM. For as $N$ is increased and no further deviations are recorded, the probability distribution will asymptotically conform to the theoretical pattern.

At this stage one might advance the argument that a zero PDF at a point $X$ on the screen means that the photon passed through both slits and interfered destructively with itself at $X$, making it impossible for the photon to reach $X$. This argument requires the assumption that the photon is 'really' a wave, from the slits all the way up to the screen, with the wavefunction 'collapsing' at the screen in order to enable the detection of the photon as a particle. But such an assumption is at best an axiom that is not provable in QM. The assertion that a photon that is fired at the slits as a particle mysteriously transforms itself into a wave, passes through both slits, and equally mysteriously ends up as a particle at the screen, has no credibility when interpreted as a 'reality'. In any case the axiomatic nature of this assertion with respect to QM means that Remark 1 still holds. For there is no proof that the same observed probability distribution cannot be arrived at by other, less mysterious routes. In summary, if one interprets the interference pattern at the screen strictly as reflecting a probability distribution for particle-like photons, and if one views the quantum formalism as merely one possible algorithm for deriving it, one cannot infer any 'reality' for the wave nature of the photon, as demonstrated by Remark 1. Young's two-slit experiment, by itself, does not prove the wave nature of the photon. One can at best assert that no photon has so far been experimentally detected at the theoretical minima of the interference pattern. But that argument does not constitute a proof of the theoretical impossibility of such a detection in all future experiments, or for all future times in a given experiment, which would be required to establish the wave nature of the photon. Indeed, such an infinitary proposition can never be 'proven' experimentally and must be axiomatic with respect to QM. Of course, in the event of such an axiomatic assertion being 
added to QM, the wave nature of the photon (which can then be inferred) must be formulated as a classically possible phenomenon rather than as a contradiction of the form $P \& \neg P$; as observed in Sec. 4.2, this will require a modification of Definition 3, and consequently, NAFL will require the violation of BCP (Definition 1) via a nonclassical model $\mathcal{N}$ in which the photon is neither a particle nor a wave. We do not believe that this is the correct approach, not only because of the mystery associated with wavefunction collapse, but also in the light of Inference 1 in Afshar's experiment. Inference 2 must be sacrificed, as will be argued below.

Next consider the NAFL interpretation, with Definition 3 in place. Let $X$ be a point located on a dark fringe in Young's two-slit experiment. To 'prove' that a photon leaving the slit(s) can never reach $X$, the standard QM formalism proceeds as follows.

(1) Assume that the photon reaches $X$ on the electronic screen.

(2) Assume $P$, assume $\neg P ; P \& \neg P$ follows. The photon takes all available paths to the point $X$.

(3) Associate a probability wave with the photon, in accordance with the standard formalism.

(4) Conclude destructive interference at $X$. The photon has a zero probability density function at $X$.

(5) Conclude from step 4 that step 1 is false, and the photon can never reach $X$ on the electronic screen.

We already questioned step 5 in the preceding analysis; a vanishing PDF does not imply proof of impossibility. Even if step 5 is granted, the above proof is not valid in the NAFL version of QM, whose rules of inference are classical. For step 2 assumes a contradiction of the the form $P \& \neg P$. Classically, any proposition can be inferred from a contradiction, and a proof based on such an inference has no validity. Therefore the conclusion (step 5) cannot be established as a theorem of QM based on the above 'proof', either in NAFL or in classical logic. Indeed, the wave nature of the photon is now classically impossible to prove as a theorem of QM, since it has been formulated as a contradiction. This holds in NAFL as well; a proposition of the form $P \& \neg P$ is not even legitimate in the theory syntax of the NAFL theory QM (see Sec. 3.4), and so cannot be a theorem. Further, $P \& \neg P$ cannot be added as an axiom to QM, say, at time $t_{1}$, for the purpose of obtaining an interpretation $\mathrm{QM}^{*}$ that retroactively asserts the wave nature of the photon, in the same manner that $Q$ was added at time $t_{2}$; as noted in Sec. 3.4. only propositions that are legitimate in the theory syntax of QM can be so added. However, it follows from Proposition 1 that consistency of QM requires the existence of a nonclassical model $\mathcal{M}$ for QM in which $P \& \neg P$ is nonclassically true. The nonclassical interpretation of $P \& \neg P$ in $\mathcal{M}$ was extensively discussed in Secs. 4.1 and 4.2, The model theory TM for $\mathcal{M}$ must be based on a paraconsistent logic (see Sec. 3.1), for TM must prove $P \& \neg P$. In such a paraconsistent logic, the classical result that any proposition 
follows from a contradiction does not hold. Therefore steps 14 in the above proof can be validly formulated in TM. Rather than asserting that the photon took all available paths to the dark spot $X$ on the screen and destructively interfered with itself, we may simply conclude that the photon did not take any path to $X$ (and indeed, is very unlikely to do so, via the proof in TM); hence the dark spot. Thus we have a setting in which the paraconsistent theory TM can, in principle, justify the standard quantum formalism in a nonclassical NAFL model $\mathcal{M}$ for QM. But note that the theorems of QM, as well as those of its interpretation $\mathrm{QM}^{*}$ (which generates $\mathcal{M}$ ), must also be theorems of TM; the contradictions provable in TM must involve only undecidable propositions of $\mathrm{QM}^{*}$, such as, $P$. In particular, since infinite sets cannot exist in NAFL theories 18 , including QM, it follows that TM cannot permit these either. One must develop real analysis in NAFL without infinite sets 18 , so that one can justify the quantum formalism in TM. One might ask, why bother with NAFL at all? Why not just use a paraconsistent logic to begin with, and just develop the theory TM? The problem is that in such an eventuality, we do not have any logical principles by which we determine which are the contradictions that should be provable in TM. For example, there would be no particular reason for $\mathrm{BCP}$ to hold; without the guiding principles of NAFL, a paraconsistent logic might as well prove the contradiction that the photon is both a wave and a particle at the same time. Thus we would be reduced to using our arbitrary intuitions rather than the principles of logic in determining what should and should not be provable. One may think of TM as essentially a set of metamathematical rules that tell us how to combine various classical models of QM so as to generate the desired nonclassical model $\mathcal{M}$ that conforms with the observer's interpretation $\mathrm{QM}^{*}$ (which in turn conforms with his observations/axiomatic declarations in the real world). Note that TM tries to predict the results of the 'measurements' made by the observer in the real world, via probabilistic reasoning. TM essentially tells us what $\mathrm{QM}^{*}$ will probably look like if the observer keeps his axiomatic declarations in $\mathrm{QM}^{*}$ in tune with his (statistically large number of) observations in the real world.

In summary, our thesis is that the observed interference pattern at the screen in Young's two-slit experiment is a manifestation of a particular nonclassical NAFL model $\mathcal{M}$ for $\mathrm{QM}$ in which the count rate of the photon at each point of the screen is in tune with the nonclassical probability distribution derived in the theory TM of $\mathcal{M}$. There could be other nonclassical NAFL models for QM that follow different probability distributions, but these may not be relevant to our real world. The important achievement here is that we have conceptually justified the use of $P \& \neg P$ in deducing the interference pattern without conceding any physical reality for the wave nature of the photon. As noted earlier, $P \& \neg P$ in $\mathcal{M}$ merely reflects the observer's ignorance of the path information of the photon and is used only for the purpose of deriving the probability distribution; the contradictions in the paraconsistent structure $\mathcal{M}$ have no 'physical' reality. To the various bright spots on the screen, each individual photon 'really' takes only one path. The only mystery here is 
that this 'reality' is not revealed via a classical probability distribution when a large number of photons are fired at the screen; instead, the resulting interference pattern revealed by Nature seems to indirectly confirm, via a nonclassical probability distribution, the observer's ignorance of the path information. Why does Nature act in this manner? The answer may have something to do with the paradoxical nature of probability, which is, even classically, a problematic notion that is dependent on the information available to the observer (hence, 'conditional' probabilities). While the photon passes through only one slit at a time as a particle, the status of the other inactive slit (i.e., whether it is open or closed) seems to impose some sort of conditionality on the probability distribution. Perhaps formulation of real analysis in NAFL, development of consistent postulates for QM, and then developing the paraconsistent theory TM of $\mathcal{M}$ will shed further light on this mystery. Many of the concepts of standard quantum theory, such as, the notion of probability, will not be formalizable in the NAFL theory QM and will have to be dealt with in TM, and in general, at a metamathematical level. This is understandable, since these concepts may be specific to the real world and the model $\mathcal{M}$ which applies to it. It is also possible that radically new concepts of space, time, and in particular, light, may be needed for a more satisfactory description of Nature in a NAFL theory that does away with probability altogether.

Let us now revert to Afshar's experiment. Afshar 1 argues that if a classical probability distribution had applied, the wire grid at location $\sigma_{1}$ in Fig. 1 should have blocked about $6.6 \%$ of the photons. Instead, the wire grid blocked fewer than $0.1 \%$ of the photons, as expected from the probability distribution calculated using the standard quantum formalism. Let us grant Afshar's argument and concede that the interference pattern does indeed exist. As argued above, this does not legitimize Inference 2, In the light of the observation of $Q$ and Inference 1, one concludes that the photon still has a metalogical particle nature for $t_{0} \leq t<t_{2}$, but with a nonclassical probability distribution corresponding to the interference pattern, as deducible in a nonclassical model $\mathcal{M}$ for QM. In particular, there was no 'destructive interference' at the dark fringes corresponding to the locations of the wires; the photons that ended up at the images simply missed the wires and the dark fringes.

Note that the axiomatic nature of NAFL truth allows the observer to axiomatically declare, via a choice of $\mathrm{QM}^{*}=\mathrm{QM}+P$ at time $t_{0}$ in Fig. 1. that a given photon passed through slit $\mathrm{U}$, even though such a measurement was not made at $t=t_{0}$. Thus the observer breaks with the informal convention of keeping his axiomatic declarations in tune with his measurements, and generates a classical particle model of the photon for $t_{0} \leq t<t_{2}$, instead of the nonclassical model $\mathcal{M}$. If at $t=t_{2}$, the photon is found to end up at the image $\mathrm{U}^{\prime}$, this would vindicate the observer's choice made through guesswork and free will. Thus in principle, NAFL allows the observer to bring the particle nature of the photon within the scope of its semantics, even though in practice, continued success on this front would require improbable guesswork on the part of the observer. Of course, consistency of QM requires $\mathcal{M}$ to 
exist (see Proposition 1), even if the observer does not choose it in any particular instance.

\subsection{Critique of Kastner's argument}

Kastner $\frac{617}{6}$ has criticized the interpretation of Inference 1 as retroactively asserting the particle nature of the photon. She has essentially two reasons for her reservations, as stated below.

- According to Kastner, the photon exhibited wave nature at time $t_{0}$ in Fig. 11in the sense that it 'really' passed through both slits, as confirmed by Inference 2 (which she supports with the caveat that the wave nature of the photon, being real, is not to be formulated as a contradiction of the form $P \& \neg P)$. So, as was noted in Sec. 4.2, Kastner requires Inference 1 to be limited to the assertion that the photon was post-selected in the state of slit $\mathrm{U}$, without any retroactive implication that the photon 'really' passed through only slit U (as a particle). In other words, Inference 1 does not provide 'which-way information' for the photon.

- Kastner cites Cramer's Transactional Interpretation (TI) of quantum mechanics 19 as unambiguously showing that the photon was selected in the superposed state of both slits at time $t_{0}$, and also was post-selected in the state of slit $\mathrm{U}$ at time $t_{2}$. However, there are intermediate times in Fig. 1, when the photon was between the locations $\sigma_{1}$ and $\sigma_{2}$, during which the ontological state of the photon is ambiguous according to TI (see Fig. 3 of Ref. 6). Kastner states that at these locations, the 'offer wave' of TI shows the photon to be in a superposition state of both slits, while the backwards-in-time 'confirmation wave' of TI shows the photon to be in a state of slit U. For this reason, Kastner asserts that it would be wrong to retroactively infer at time $t_{2}$ that the photon passed through only slit $\mathrm{U}$, since such an inference would require the photon to be determinately a particle at all intermediate locations between $\sigma$ and $\sigma_{2}$ in Fig. 1 .

On the basis of the above criticisms, Kastner concludes that Afshar's experiment does not refute BCP (Definition 1).

We have exhaustively addressed the problems with the first of Kastner's criticisms. From the point of view of NAFL, Inference 1 is merely the observer's retroactive axiomatic declaration of the particle nature of the photon. But the concept of 'axiomatic declaration' itself cannot be formalized in the theory syntax of NAFL theories 15 . So when the observer 'post-selects' the photon as being in the 'state of slit U', he can only have in mind that the photon 'really' passed through slit U as a particle. Therefore, in NAFL, the classical model of QM that is generated at time $t_{2}$ via the observer's interpretation $\mathrm{QM}^{*}=\mathrm{QM}+Q$, must reflect such a retroactive implication for Inference 1. In fact, the completeness theorem of first-order logic (which is a metamathematical principle in NAFL) requires that there must exist 
such a classical model for QM. For, as we have already pointed out, QM does not prove either $\neg P$ or Kastner's claim that the photon passed through both slits at time $t_{0}$. This latter claim, affirming the wave nature of the photon at time $t_{0}$, is at best another metamathematical requirement that Kastner chooses to impose. In the conflict between these two metamathematical requirements, the completeness theorem wins out in NAFL; it is a sacred principle of logic that cannot be sacrificed. On the other hand, the nonclassical NAFL model $\mathcal{M}$ of QM that affirms $P \& \neg P$ at time $t_{0}$ does not conflict with the above retroactive assertion of $P$, either temporally or in meaning, as we have already pointed out in Secs. 4.1 and 4.2 . In particular, $\mathcal{M}$ only affirms the observer's lack of information on the path of the photon at time $t_{0}$, rather than Kastner's assertion that the photon 'really' passed through both slits.

The second of Kastner's objections is also problematic, in the sense that her stated purpose of rescuing BCP is not served by her invocation of Cramer's TI. If TI requires that the photon's ontological state is ambiguous between the locations $\sigma_{1}$ and $\sigma_{2}$ in Fig. 1 that amounts to a violation of BCP when the photon was at these locations. For we have determined, using TI, that the photon was neither a particle nor a wave when it was at these locations. So the NAFL model of QM that applies at these times would have to be a superposition of both the particle and wave states of the photon, i.e., a superposition of a classical and a nonclassical model of QM. But such a superposition of models does not exist in NAFL and clearly violates BCP; see Definition 1 and also the NAFL version of BCP as stated at the end of Sec. 4.2. This is not surprising, for TI requires us to ascribe reality to the superposed state of the photon passing through both slits; this is also Kastner's belief, as noted above. As a consequence, NAFL would require that Definition 3 be modified to force, via Proposition 11 the existence of a nonclassical model $\mathcal{N}$ for QM that violates BCP (as was pointed out in Sec. 4.2).

\subsection{Critique of Cramer's TI in the delayed-choice scenario}

One example of a quantum mystery arises from the well-known delayed choice experiment of Wheeler 20. In Fig. 1, after a photon passes through the slit(s) in the $\sigma$ plane at time $t_{0}$, the observer could either choose to insert a screen in the $\sigma_{1}$ plane, or he could choose to allow the photon to pass through the lens and reach the $\sigma_{2}$ plane. So according to Wheeler, this delayed choice of measurement means that the observer could post-select (i.e., after time $t_{0}$ ) each photon to pass through both slits, as a wave, or one slit only, as a particle. But this amounts to choosing one's past after the event and seems paradoxical. In the NAFL interpretation, the metalogical reality of the photons as particles means that the delayed choice does not influence the past; each photon always passed through one and only one slit. The interference pattern, which one sees on the screen after many photons are recorded on it, also exists (but is not measured) for the photons that end up at the $\sigma_{2}$ plane, as is spectacularly confirmed in Afshar's experiment. 
Cramer's Transactional Interpretation (TI) 19, on the other hand, provides an explanation for delayed choice by positing an 'atemporal' transaction that takes place between 'offer waves' and 'confirmation waves', with the latter propagating backwards in time. From the point of view of NAFL, however, which rejects the relativistic conception of 'spacetime' (see Appendix B of Ref. 17), such an atemporal transaction is not physically possible, and neither can anything propagate backwards in time. The temporal and axiomatic nature of NAFL truth requires absolute time, as well as Euclidean space. Cramer's aphysical approach to delayed choice really amounts to taking an anti-realist stand that the 'past' exists if and only if, and only after, it is clearly defined. This is confirmed by Cramer's assertion that "No offer is a transaction until it is a confirmed transaction", which corresponds to Wheeler's "No phenomenon is a phenomenon until it is an observed phenomenon". Such an anti-realist stand is possible in NAFL by associating 'measurement' or 'observation' with 'axiomatic declaration', and denying any metalogical reality outside of NAFL syntax and semantics (as was noted in Sec. 4.2). But in NAFL, the superposed state is not really a physical state of the photon passing through both slits, as is assumed by Cramer; the anti-realist stand, when imposed upon the NAFL interpretation, would further require that the photon has no determinate state in the real world when it is deemed to be in a quantum superposition of passing through both slits. Hence from the point of view of NAFL, Cramer's TI is not consistent with either realism or anti-realism. As was discussed in Sec. 4.2 positing a metalogical reality for the particle nature of the photon is not only compatible with NAFL, but is also philosophically a more satisfactory resolution of the paradoxes associated with Afshar's experiment. From this realist point of view, Cramer's TI, by positing waves propagating backwards in time, seems to accept that one can influence the past; this is denied in the NAFL interpretation as aphysical. In Sec. 4.4 we have criticized Kastner's 6 defence of BCP, using Cramer's TI, as logically problematic; the said defense upholds $\mathrm{BCP}$ at the slits by requiring its violation elsewhere, at least according to the NAFL interpretation.

\section{The coin toss and Schrödinger cat experiments}

Consider the coin toss experiment described in Sec. 2.5 of Ref.16. An observer tosses a fair coin and, as it lands at time $t_{0}$, covers the coin under the palm of his hand without seeing the outcome. Let $P(\neg P)$ represent "The outcome is 'heads' ('tails')". Let the observer have the NAFL theory $\mathrm{T}$ in mind, which includes definitions describing this coin toss experiment. Further, at $t=t_{2}$, the observer lifts his hand and sees the outcome, say, 'heads'. For $t_{0} \leq t<t_{2}$, the observer chooses the interpretation $\mathrm{T}^{*}=\mathrm{T}$. Hence $P \& \neg P$ holds for the observer in a nonclassical model $\mathcal{T}$ for $\mathrm{T}$, signifying that he has not measured (axiomatically declared) the outcome to be either 'heads' or 'tails' during this time interval. Let $R$ denote the proposition that "The outcome is 'heads' for $t \geq t_{2}$ ". For $t \geq t_{2}$, the observer takes $\mathrm{T}^{*}=\mathrm{T}+R$, in tune with his observation, so that $P$ (which is provable in $\mathrm{T}^{*}$ for these times) 
holds in a classical model for $\mathrm{T}$. Let the proposition $Q$, formulated for $t \geq t_{2}$, denote "The outcome was 'heads' during $t_{0} \leq t<t_{2}$ ". Our contention is that the theory $\mathrm{T}^{*}=\mathrm{T}+R$ proves $Q$, so that the observer has retroactively asserted at $t=t_{2}$ that the outcome was always 'heads' during $t_{0} \leq t<t_{2}$.

Is the inference of $Q$ in $\mathrm{T}+R$ legal? In this case, the observer can feel confident that the outcome 'heads' must be metalogically ('really') true for $t_{0} \leq t<t_{2}$. The observer knew that the coin was flat under the palm of his hands during $t_{0} \leq t<t_{2}$, but as was noted in Sec. 2.5 of Ref. 16, this fact, being provably equivalent to $P \vee \neg P$ in the proof syntax of $\mathrm{T}$, cannot be formalized as a legal proposition in the theory syntax of T. Such an (illegal) formalization would force $P \vee \neg P$ to be a theorem of $\mathrm{T}$, and prevent the existence of the nonclassical model $\mathcal{T}$ required by Proposition 1 for consistency of $\mathrm{T}$ in NAFL. The reason this example is interesting is that the observer knows that the superposed state of 'heads and tails' in $\mathcal{T}$ has no 'physical' reality, but nevertheless it correctly reflects the observer's ignorance of the outcome during $t_{0} \leq t<t_{2}$. In Afshar's experiment, the situation is logically similar, but the observer does not have such a clear intuition for the particle nature of the photon, and consequently, for the validity of Inference 1. But nevertheless, NAFL treats both cases similarly and in a logically consistent manner.

Next reconsider the Schrödinger cat experiment described in Sec. 3.3. Once again the observer has the intuition that the retroactive assertion of $U$, via an inference in the theory $\mathrm{QM}^{*}=\mathrm{QM}+V$, is true in the real world. This inference is based on the principle (deducible in the proof syntax of $\mathrm{QM}^{*}$ ) that if one finds the cat to be alive at time $t_{2}$ and if one knows that the cat was alive at an earlier time $t_{0}$, then the cat was alive for $t_{0}<t<t_{2}$. Thus at $t=t_{2}$, we know that the cat was (metalogically, 'really' and unambiguously) alive during $t_{0}<t<t_{2}$. NAFL supports such a rock-solid, unimpeachable inference that conforms with the standard definition of 'alive'.

\section{Concluding Remarks}

The NAFL interpretation upholds the Bohr complementarity principle (BCP) in Afshar's experiment $1 / 2 \mid 3$ by retroactively affirming the 'real' particle status of the photon, even while semantically the photon was in a superposed state $(S)$ in a nonclassical model $\mathcal{M}$ of the NAFL theory QM formalizing quantum mechanics. However, such a 'reality' for the particle state, which is outside of both the syntax and the semantics of QM, can be said to be metalogical. In NAFL, $S$ (or the "wave nature' of the photon) only reflects the fact that the observer has not measured (axiomatically asserted) the true, classical path of the photon; no physical reality, to the effect that the photon 'really passed though both slits and interfered with itself', can be assigned to $S$. The interference pattern in the $\sigma_{1}$ plane of Fig. 1 must be interpreted in NAFL as reflecting a nonclassical probability distribution for the photons, still treated as particles, that is derivable within $\mathcal{M}$. We have no explanation yet for why the lack of which-way information influences the probability 
distribution in this manner.

The still nascent NAFL interpretation of quantum mechanics has considerable potential for future research. The subtle formulation of the syntax and semantics of NAFL theories to combine both classical and intuitionistic principles, the ability of NAFL to handle the temporal nature of mathematical truth, and the demonstration of the need for a paraconsistent logic to handle the model theory of NAFL theories are features that make the NAFL interpretation highly suitable for the purpose of providing a logical explanation for the mysteries of quantum mechanics, within the framework of a single logic. This, despite the limitations that NAFL imposes on classical infinitary reasoning $15|16| 17 \mid 18$, is a great advantage of The NAFL interpretation, which can be said to provide a logical basis for many of Niels Bohr's great physical ideas that were spelt out in the Copenhagen interpretation. It is to be emphasized the NAFL interpretation is not essential for the purpose of explaining the results of the Afshar experiment and other seemingly paradoxical experiments of quantum mechanics; the Copenhagen interpretation is adequate for this purpose. However, at present quantum mechanics cannot be satisfactorily formalized within a single logic. For example, real analysis uses the framework of classical logic while other quantum phenomena like superposition require nonclassical logics. If the NAFL interpretation can be developed to its full potential, a formalization of all of quantum mechanics within a single logic would hopefully be within reach.

\section{References}

1. S. S. Afshar, E. Flores, K. F. McDonald, E. Knoesel, Paradox in wave-particle duality, Foundations of Physics 37 (2007) 295-305.

2. S. S. Afshar, Violation of the principle of complementarity, and its implications, in The Nature of Light: What is a Photon, eds. C. Roychoudhuri and K. Creath, Proc. SPIE 5866 (2005) 229-244. Preprint version available at: http://www.irims.org/quant-ph/030503/.

3. S. S. Afshar, Violation of Bohr's complementarity: one slit or both?, AIP Conference Proceedings 810 (2006) 294-299. Eprint: arXiv:quant-ph/0701039.

4. D. M. Greenberger, A. Yasin, Simultaneous wave and particle knowledge in a neutron interferometer, Phys. Lett. A 128 (1988) 391-394.

5. B. G. Englert, Fringe visibility and which-way information: an inequality, Phys. Rev. Lett. 77 (1996) 2154-2157.

6. R. E. Kastner, Why the Afshar experiment does not refute complementarity, Studies In History and Philosophy of Science Part B: Studies In History and Philosophy of Modern Physics 36 (2005) 649-658. Eprint: arXiv:quant-ph/0502021

7. R. E. Kastner, On visibility in the Afshar two-slit experiment, Foundations of Physics 39 (2009) 1139-1144. Eprint: arXiv:0801.4757 [quant-ph].

8. T. Qureshi, Complementarity and the Afshar experiment, arXiv:quant-ph/0701109.

9. D. Reitzner, Comment on Afshar's experiments, arXiv:quant-ph/0701152.

10. A. Drezet, Complementarity and Afshar's experiment, arXiv:quant-ph/0508091

11. O. Steuernagel, Afshar's experiment does not show a violation of complementarity, arXiv:quant-ph/0512123.

12. R. Srikanth, Physical reality and the complementarity principle, arXiv:quant-ph/0102009 
13. E. Flores, E. Knoesel, Why Kastner analysis does not apply to a modified Afshar experiment, arXiv:quant-ph/0702210.

14. P. O'Hara, Entanglement and quantum interference, arXiv:quant-ph/0608202

15. R. Srinivasan, The quantum superposition principle justified in a new non-Aristotelian finitary logic, International Journal of Quantum Information 3 (2005) 263-267; Proc. Foundations of Quantum Information, University of Camerino, Italy, April 16-19 (2004). Preprint available at: http://philsci-archive.pitt.edu/archive/00001923/ .

16. R. Srinivasan, Platonism in classical logic versus formalism in the proposed nonAristotelian finitary logic, Philosophy of Science Archive, Preprint ID Code 1166. Available at: http://philsci-archive.pitt.edu/archive/00001166/.

17. R. Srinivasan and H. P. Raghunandan, On the existence of truly autonomic computing systems and the link with quantum computing, arXiv:cs.LO/0411094

18. R. Srinivasan and H. P. Raghunandan, Foundations of real analysis and computability theory in non-Aristotelian finitary logic, arXiv:math.LO/0506475

19. J. G Cramer, The Transactional Interpretation of Quantum Mechanics, Rev. Mod. Phys. 58 (1986) 647-688.

20. J. A. Wheeler, The "past" and the "delayed-choice" double-slit experiment, in Mathematical Foundations of Quantum Theory, ed. A. R. Marlow (Academic Press, New York, 1978), pp. 9-48. Reprinted in Quantum Theory and Measurement, eds. J. A. Wheeler and W. H. Zurek (Princeton University Press, New Jersey, 1983), pp. $182-213$.

21. M. O. Scully, Y. H. Kim, R. Yu, S. P. Kulik and Y. H. Shih, A delayed-choice quantum eraser, Phys. Rev. Lett. 84 (2000) 1-5.

22. G. N. Mardari, An alternative to quantum complementarity, arXiv:quant-ph/0409197. 